Supplement of

\title{
Comparison of statistical downscaling methods for climate change impact analysis on drought
}

Hossein Tabari ${ }^{1}$, Daan Buekenhout ${ }^{1}$, Patrick Willems ${ }^{1,2}$

${ }^{1}$ KU Leuven, Department of Civil Engineering, Belgium.

${ }^{2}$ Vrije Universiteit Brussel, Department of Hydrology and Hydraulic Engineering, Belgium.

Correspondence to: Hossein Tabari (hossein.tabari@kuleuven.be)

This PDF file includes:

Texts S1 to S2

Figures S1 to S6 


\section{Text S1. Fitting two-component mixed exponential distributions}

SDM4 requires fitting two-component mixed exponential functions to precipitation values and to dry event durations.

The density function of a two-component mixed exponential distribution can be written as:

$f\left(x ; p_{1} ; p_{2} ; \lambda_{1} ; \lambda_{2}\right)=p_{1} \lambda_{1} e^{-\lambda_{1} x}+p_{2} \lambda_{2} e^{-\lambda_{2} x}$

Typically, mixed exponential distribution is constructed as a weighed sum of the exponential distribution. This simplifies Eq. S1 to:

$f\left(x ; p ; \lambda_{1} ; \lambda_{2}\right)=p \lambda_{1} e^{-\lambda_{1} x}+(1-p) \lambda_{2} e^{-\lambda_{2} x}$

The cumulative density function of two-component mixed exponential distributions can consequently be written as:

$F\left(x ; p ; \lambda_{1} ; \lambda_{2}\right)=p\left(1-e^{-\lambda_{1} x}\right)+(1-p)\left(1-e^{-\lambda_{2} x}\right)$

A popular way to fit this type of distribution to data points is by application of iterative Expectation-Maximization algorithms (Yilmaz et al., 2015). An implementation of this algorithm for fitting mixed exponential distributions is included in the R package 'Renext' (Deville and IRSN, 2016). This package also includes other useful functions related to mixed exponential distributions, e.g. for generating random samples.

Figure S1 shows the two-component mixed exponential density functions that are fitted to the empirical probabilities of observed (Uccle) dry event lengths for SDM3. The fitted distributions underestimate the proportion of inter-events with a duration of 1 day. This underestimation is countered when sampling since the complete range $[0,1.5]$ of sampled durations is rounded to 1 day. Figure $\mathrm{S} 2$ shows the two-component mixed exponential cumulative density functions that are fitted to the seasonal empirical cumulative density functions of observed (Uccle) daily precipitation intensities for SDM3. The fitted distributions are very close to simple exponential distributions since $p_{1} \approx p_{2} \approx 0.5$ and $\lambda_{1} \approx \lambda_{2}$. 

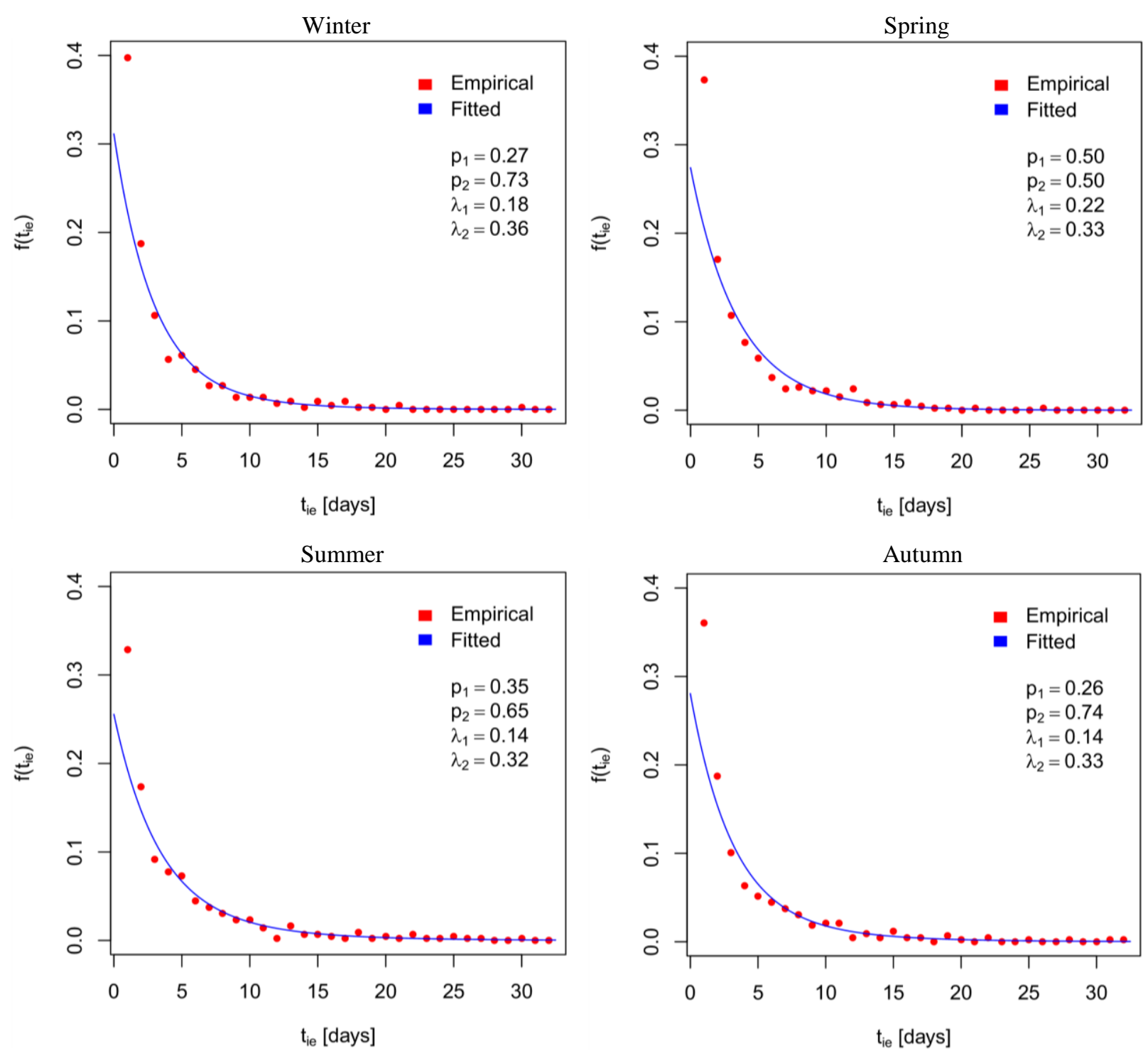

Figure S1. Fitted two-component mixed exponential distributions to seasonal empirical probabilities of observed (Uccle) dry event durations. 

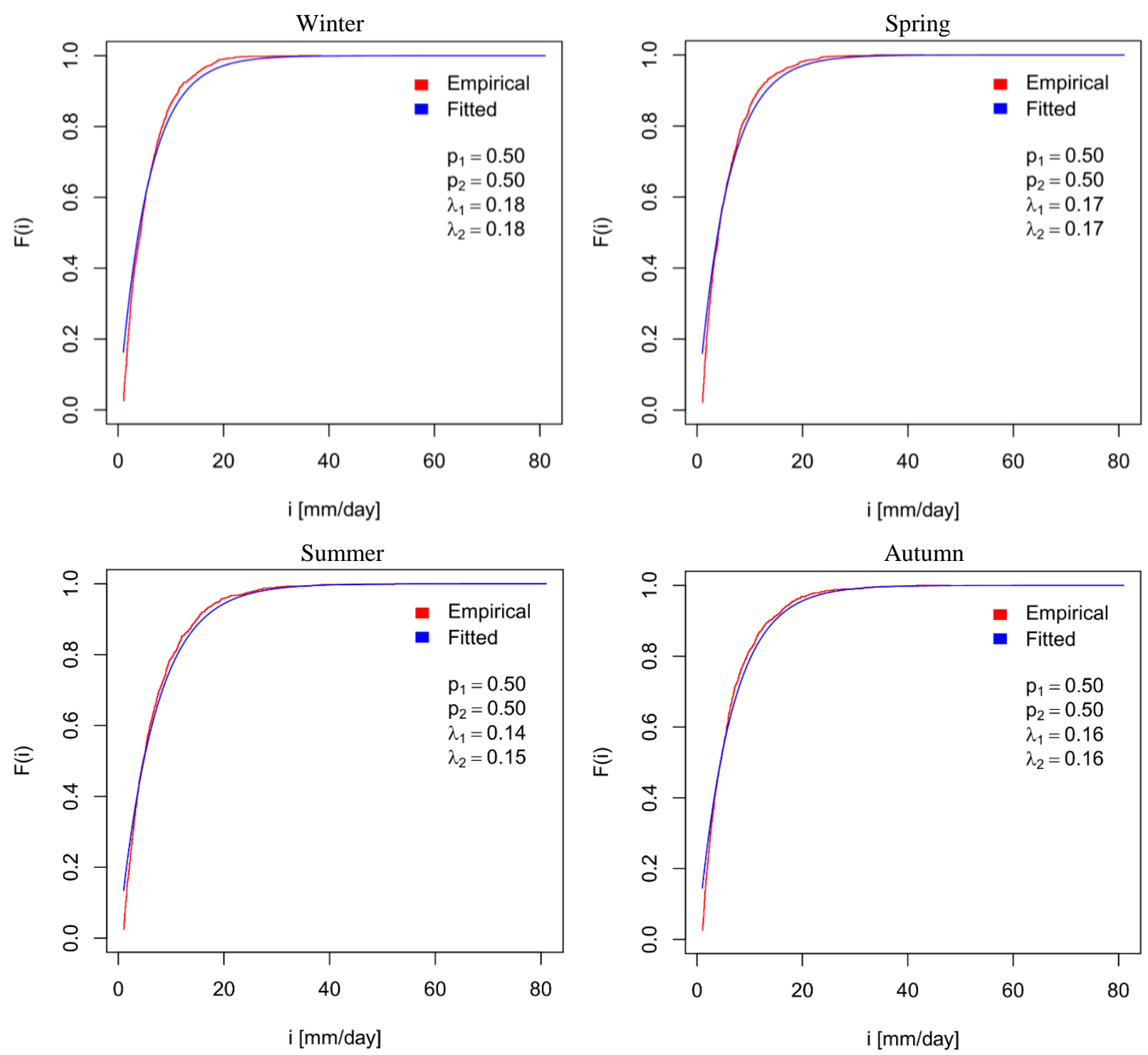

Figure S2. Fitted two-component mixed exponential distributions compared to empirical cumulative density functions of seasonal observed (Uccle) daily precipitation intensities. 


\section{Text S2. Sensitivity analysis}

In order to gain insight in the downscaling methods, tests can be performed by altering the method's input parameters. Sensitivity analyses are performed on relevant parameters for SDM3 and SDM4. Attention is given to the number of simulations and the corresponding results. For SDM4, the sampling range for the parameters of the dry spell distributions is also of interest.

\section{Sensitivity analysis for SDM3 ( $Q P$ method)}

In a QP downscaling method, the state (wet or dry) of days in the original time series can be converted in order to match the projected changes in dry day frequency better. Different strategies exist for the selection of the day subject to conversion. The SDM3 implementation makes use of a Markov chain process in which the stochastic component is handled by executing multiple simulations to select the 'best'. In this sensitivity analysis, the QP method is tested for varying numbers of simulations (i.e., 10, 100 and 1000). The objective is to uncover to what extent a significant increase in the number of simulations impacts the results. Based on this, a choice can be made on the number of simulations that should be used in this research. The computer processing time increases with the number of simulations but remains within the order of minutes for all three tests. For this analysis, the 14-member CMIP6 GCM ensemble for the high-end SSP5-8.5 scenario is selected.

Upon increasing the number of simulations, the number of dry days per month (NDD $\mathrm{m}$ ) remains constant (Figure S3). This result is expected because every simulation has been constructed by the QP method to achieve the same projected monthly dry day frequencies. As a consequence, a larger number of simulations has no effect on these dry day indicators. A different image is painted by the RIs describing very long dry spells (VLDS). The number (NDS 5 ) and the median length (DSL) of the dry spells in this dry spell class evolve without a clear pattern, as illustrated by Figure S3. There is no sign of convergence upon increasing the number of simulations.

The mean daily maximum precipitation per month is displayed in Figure S4 for the months of January and July. The latter month shows a pattern of larger changes upon altering the number of simulations. Based on the monthly graphs (not shown), it cannot be generalized that the summer months show larger changes than the winter months or the other way around. Again, the graphs do not suggest a converging trend upon increasing the number of simulations.

A different result is found for the mean monthly precipitation, as shown in Figure S5. The changes resulting from the alteration of the number of simulations are of a lower order of magnitude than the inter-GCM differences. Unlike the dry spell related research indicators, the monthly precipitation displays a converging trend by raising the number of simulations. This is true for all months (not shown). Some relatively large corrections can be found by increasing the number of simulations from 10 to 100 . Increasing the number of simulations beyond this level has practically no further effect. 

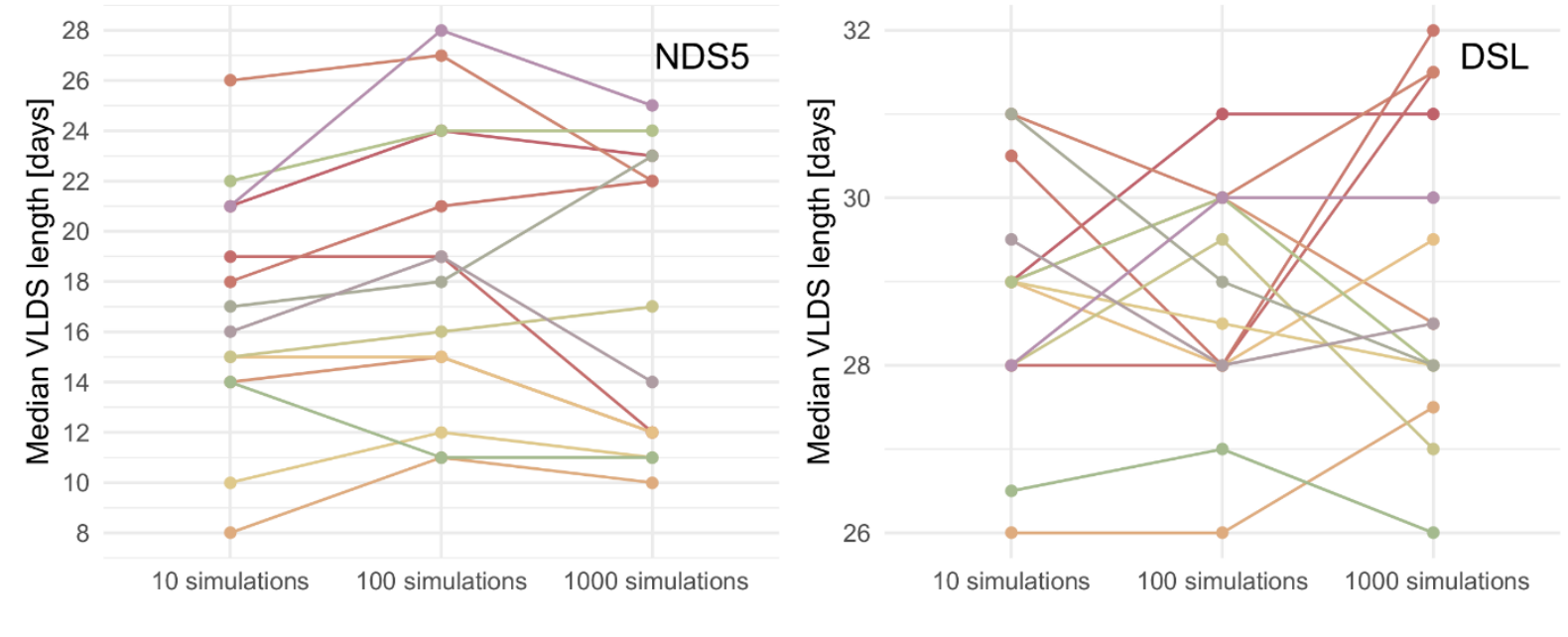

Figure S3. Evolution of dry spell related research indicators with increasing number of simulations using the QP downscaling method. Each line represents a GCM in the used ensemble. Variation in color is strictly applied to ease visual differentiation.
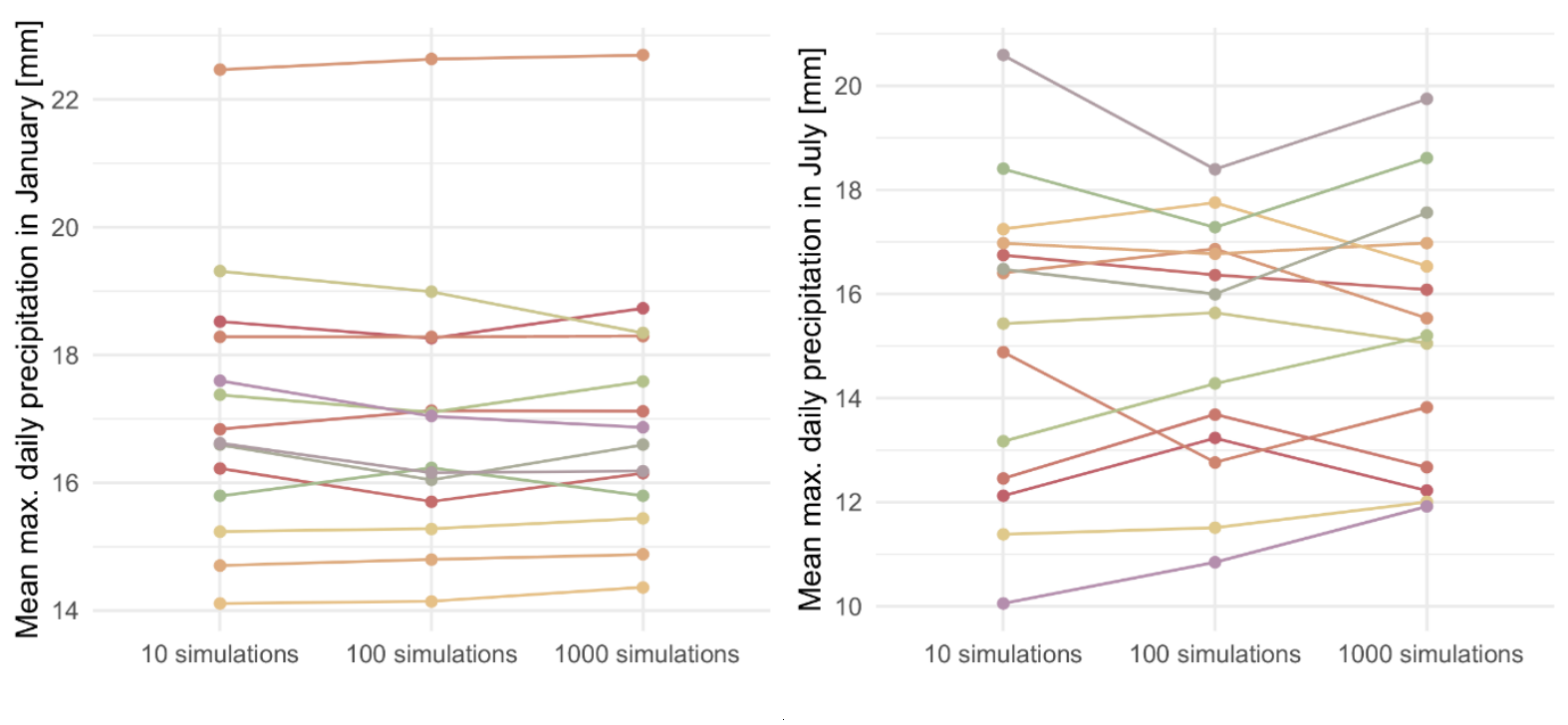

Figure S4. Evolution of the mean maximum daily precipitation per month with increasing number of simulations using the QP downscaling method. Each line represents a GCM in the used ensemble. Variation in color is strictly applied to ease visual differentiation. 

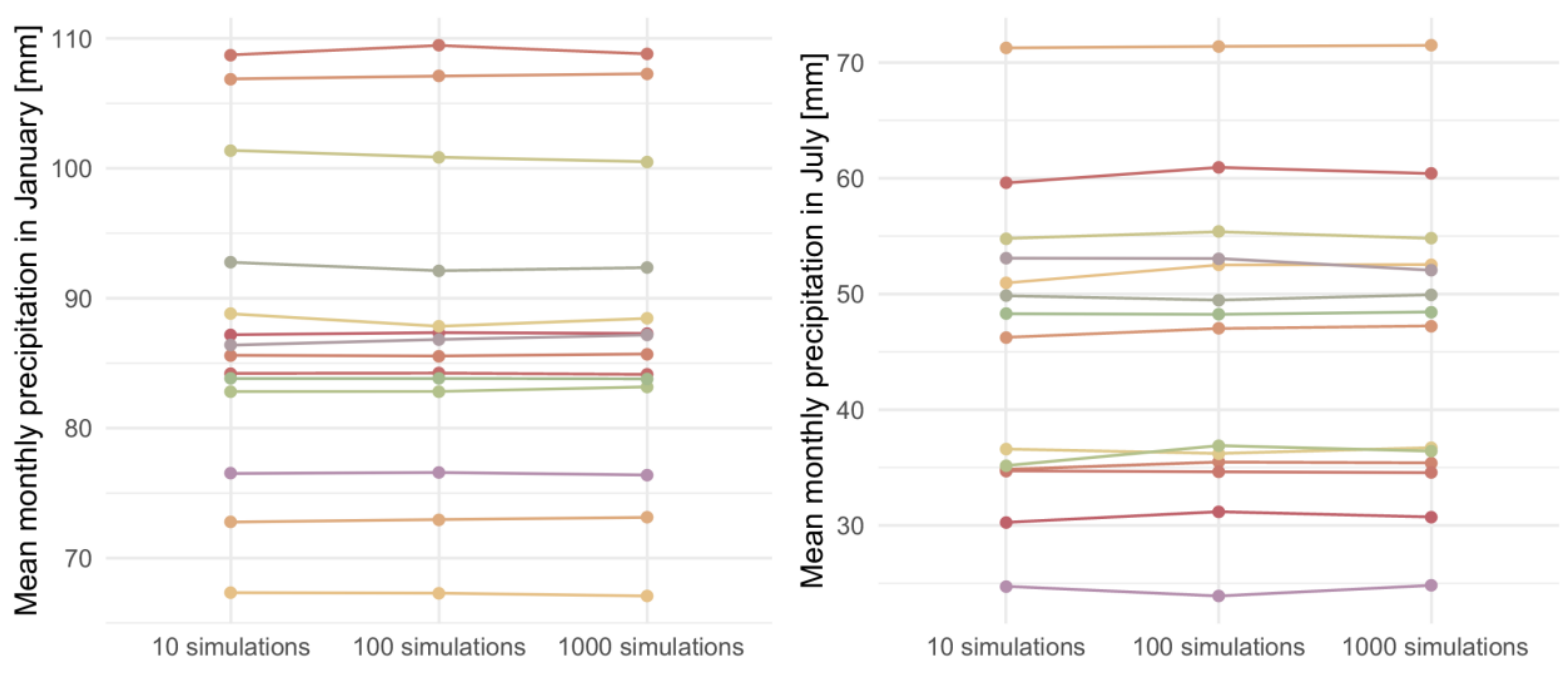

Figure S5. Evolution of the average monthly precipitation with increasing number of simulations using the QP downscaling method. Each line represents a GCM in the used ensemble. Variation in color is strictly applied to ease visual differentiation.

\section{Sensitivity analysis for SDM4 (EBWG)}

The event based weather generator (EBWG) described by Thorndahl et al. (2017) is also subject to a sensitivity analysis. For the application of this method, the user has to arbitrarily set multiple parameters. Two parameters are found relevant for a sensitivity analysis. A first parameter is the number of simulations. Similar to the QP method, multiple simulations are generated to counter for the stochastic elements contained in this method. Weather generators are stochastic by nature since they assume probability distributions for underlying components (e.g. dry event durations). Dissimilar to the QP method, the number of simulations is notably higher due to the large number of stochastic components present in the EBWG. It is important to achieve sufficiently large numbers of accepted simulations. A low number of simulations limits the pool of candidates for the selection of the 'best' simulation, which in turn corresponds to a lowered importance of the target variables' weights. 'Sufficiently large' is - arbitrarily imagined as a few hundred accepted simulations. The original implementation considers 10,000 simulations. In this analysis, this number is increased to 20,000 and 30,000 . The computer processing time for 10,000 simulations is in the order of hours.

The second parameter considered in this sensitivity analysis is related to the permissible change to the parameters of the original dry event length probability distributions (fitted to observed data). Since the main objective of this research is the investigation of the change in drought, the sensitivity analysis is performed only for the change that is made to the dry spell related distributions. The two-component mixed exponential distributions that are used to sample dry spell lengths have three parameters: the rate parameters $\lambda_{1}$ and $\lambda_{2}$ describing the two components of the distribution and $p_{1}$ representing the weighing of both components. For this sensitivity analysis, the sampling range is taken equal for all parameters and for all seasons. This range is expressed as a percentage of the original (fitted) value. The set of $3 \times 4$ parameters is consequently reduced to 1 parameter. Three different sample ranges are considered: $20 \%$, 
$30 \%$ and $40 \%$. The $20 \%$ corresponds well with the intervals used in the original implementation, which was used to downscale RCP4.5 scenario results. The remaining parameters describing wet day precipitation changes $(\alpha$ and $\beta)$ are set at fixed sampling boundaries and are not part of this analysis.

The analysis makes use of two internal indicators of the EBWG for evaluation. The first indicator is the number of simulations that are accepted. This indicator is expected to directly show the effect of enlarging the sampling intervals. A second indicator is the performance of the 'best' simulation. The analysis is applied to the 14-member ensemble of CMIP6 GCMs for all four selected climate change scenarios.

The results are summed up and visualized as heat maps in Figure S4. As expected, the number of accepted simulations scales almost perfectly with the number of simulations. Large differences in accepted simulations are observed when comparing different climate change scenarios. Performance on the other hand only appears to rise slightly by increasing the number of simulations. Performance drops notably when comparing the low level SSP1-2.6 climate change scenario with the high level SSP5-8.5 scenario.

Increasing the sampling range clearly has an effect on the number of accepted simulations. The effect for the low level SSP1-2.6 scenario is rather negative: more simulations are accepted by using a smaller sampling interval. The high level SSP5-8.5 scenario is positively impacted by a higher sampling range, resulting in more accepted simulations. The results for medium to high level scenarios (SSP2-4.5 and SSP3-7.0) show the transition between the low and high level scenarios. The performance does not display such well-defined patterns. Performance values in general are very similar within each climate change scenario.

The main difference between the low and high level climate change scenarios is the magnitude of climate changes projected by them. SSP5-8.5 projections have larger change factors than SSP1-2.6 for the considered target variables, e.g. for the seasonal number of dry days. The larger the changes, the less likely it is for the EBWG to produce a simulation that is close enough to the projections to get accepted. Consequently, there are less accepted simulations for high level scenarios. Where 10,000 simulations largely suffice for SSP1-2.6, SSP5-8.5 might benefit from increasing the number of simulations even beyond 30,000. The same rationale can be applied to explain the favorable trend of enlarging the sampling interval for the higher-end scenarios since larger sampling intervals allow simulation of larger changes. The lower-end scenarios suffer from the larger sampling intervals because changes more frequently become too large to accept.

Dry spell related target variables (i.e. seasonal number of dry days) account for $50 \%$ of the performance. The other target variables are not directly affected by a change in the dry event length distribution that is considered in this analysis. This attributes to the low fluctuations encountered for the best performance, especially within the same climate change scenario. The variation among scenarios is explained by the larger change factors of the higher-end scenarios that are harder to capture with this method. 

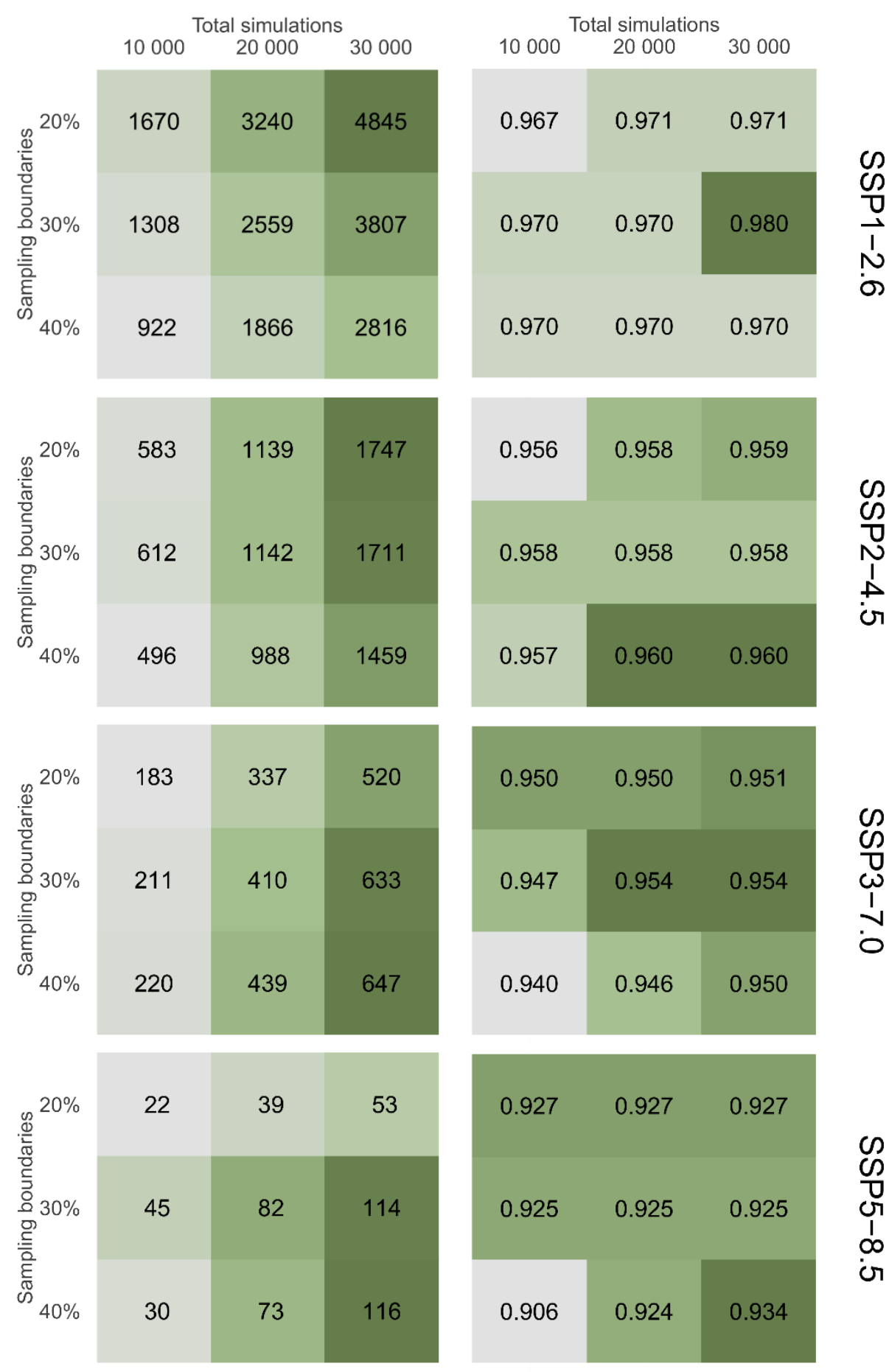

(a) Number of accepted simulations

(b) Best performance

Figure S6. Results of the sensitivity test for the EBWG (SDM 4). Low values are represented in grey, high values in dark green. The performance of a simulation is calculated using the target variables and weights listed in Table 2 . 


\section{References}

Deville, Y., and IRSN: Renext: Renewal Method for Extreme Values Extrapolation, R package version 3.1-0, 2016.

Thorndahl, S., Andersen, A. K., and Larsen, A. B.: Event-based stochastic point rainfall resampling for statistical replication and climate projection of historical rainfall series, Hydrol. Earth Syst. Sci., 21, 4433-4448, doi:10.5194/hess-21-4433-2017, 2017.

Yilmaz, M., and Buyum, B.: Parameter estimation methods for two-component mixed exponential distributions, ISTATISTIK: J. Turk. Stat. Assoc., 8(3):51-59, 2015. 